\title{
Antiplatelet drugs for prevention of pre-eclampsia and its consequences: systematic review
}

\author{
Lelia Duley, David Henderson-Smart, Marian Knight, James King
}

\begin{abstract}
Objective To assess the effectiveness and safety of antiplatelet drugs for prevention of pre-eclampsia and its consequences.

Design Systematic review.

Data sources Register of trials maintained by

Cochrane Pregnancy and Childbirth Group,

Cochrane Controlled Trials Register, and Embase.

Included studies Randomised trials involving women at risk of pre-eclampsia, and its complications, allocated to antiplatelet drug(s) versus placebo or no antiplatelet drug.

Main outcomes measures Pre-eclampsia, preterm birth, fetal or neonatal death, and small for gestational age baby. Studies were assessed for quality of concealment of allocation and losses to follow up. Results 39 trials (30 563 women) were included, and 45 trials ( $>3000$ women) excluded. Use of antiplatelet drugs was associated with a $15 \%$ reduction in the risk of pre-eclampsia (32 trials, 29331 women; relative risk $0.85,95 \%$ confidence interval 0.78 to 0.92 ; number needed to treat 100,59 to 167 ). There was also an $8 \%$ reduction in the risk of preterm birth (23 trials, 28268 women; $0.92,0.88$ to $0.97 ; 72,44$ to 200 ), and a $14 \%$ reduction in the risk of fetal or neonatal death (30 trials, 30093 women; $0.86,0.75$ to 0.98 ; 250,125 to $>10000$ ) for women allocated antiplatelet drugs. Small for gestational age babies were reported in 25 trials (20 349 women), with no overall difference between the groups (relative risk $0.92,0.84$ to 1.01 ). There were no significant differences in other measures of outcome. Conclusions Antiplatelet drugs, largely low dose aspirin, have small to moderate benefits when used for prevention of pre-eclampsia.
\end{abstract}

\section{Introduction}

Pre-eclampsia, defined as hypertension associated with proteinuria, ${ }^{1}$ complicates $2-8 \%$ of pregnancies. ${ }^{2}$ Although the outcome for most women is good, severe pre-eclampsia and eclampsia (the rare occurrence of seizures superimposed on pre-eclampsia) are important causes of maternal mortality, being associated with $10-15 \%$ of maternal deaths. ${ }^{3}{ }^{4}$ Perinatal mortality is also increased. ${ }^{5}$ There is little good quality information about maternal and perinatal morbidity, but it also is probably high. For the one in ten women who develop pregnancy induced hypertension (raised blood pressure without proteinuria), the outcome is similar to that for women with normal blood pressure. ${ }^{6}$

The cause of pre-eclampsia remains unknown, but the condition is associated with deficient intravascular production of prostacyclin, a vasodilator, and excessive production of thromboxane, a platelet derived vasoconstrictor and stimulant of platelet aggregation.? These observations led to the hypothesis that antiplatelet drugs might prevent or delay the develop- ment of pre-eclampsia. This hypothesis has been widely tested in randomised trials. Exciting results from early small trials led on to several large studies of low dose aspirin. However, the collaborative low dose aspirin study in pregnancy, the largest trial to date, failed to confirm any worthwhile benefit. ${ }^{\text {w11 }}$ Previous systematic reviews, all now out of date, concluded that although low dose aspirin seems safe, it is not effective for most women. ${ }^{8-13}$ Issues such as whether there is greater benefit for high risk women, what dose to use, and when to start treatment remain controversial, and publication bias has been suggested as a possible explanation of the discrepancy between the results of early metaanalyses and later large trials. ${ }^{14}$

This systematic review is published in full in the Cochrane Library. ${ }^{15}$ The aim was to assess the effects of antiplatelet drugs for the prevention of pre-eclampsia and its complications. We also explored the extent to which publication bias may have influenced speed and accessibility of the results of these trials.

\section{Methods}

\section{Search strategy}

The full search strategy is described elsewhere..$^{15}$ The search included the Cochrane Pregnancy and Childbirth Group register of trials, the Cochrane Controlled Trials Register, ${ }^{16}$ Embase (1994-9), and hand searching of conference abstracts. We included randomised trials comparing antiplatelet agent(s) with placebo or no antiplatelet agent for women at risk of developing preeclampsia. Quasi random designs were excluded. Main outcomes were pre-eclampsia, preterm delivery, stillbirth or neonatal death, and a small for gestational age baby.

\section{Assessment of validity}

The studies were assessed independently by two reviewers. There was no blinding of authors or results. The four of us worked in pairs, one based in the United Kingdom and the other in Australia. Differences were resolved by discussion between the pair. If differences could not be resolved, the other pair was consulted.

We assessed the validity of each included trial according to the criteria outlined in the Cochrane handbook, ${ }^{17}$ giving each trial a grade for concealment of allocation: a (adequate), b (unclear), or c (clearly inadequate). If the method of concealment was unclear, we tried to contact authors for further details. Losses to follow up were documented, and we excluded studies or outcomes for which over $20 \%$ of participants were not reported.

\section{Abstraction and synthesis of data}

We all independently extracted data from the papers, working in pairs. Discrepancies were resolved by discussion. If we could not agree, the data were excluded until further clarification was available from

\section{Resource Centre \\ for Randomised \\ Trials, Institute of Health Sciences, Oxford OX3 7LF \\ Lelia Duley obstetric \\ epidemiologist \\ Health Services Research Unit, Institute of Health Sciences \\ Marian Knight specialist registrar \\ New South Wales Centre for Perinatal Health Services \\ Research, Queen \\ Elizabeth II Institute for Mothers and Infants, Sydney, \\ Australia \\ David \\ Henderson-Smart \\ director \\ Perinatal \\ Epidemiology Unit, Mater Hospital, Brisbane, Australia James King \\ director \\ Correspondence to: L Duley \\ lelia.duley@ \\ ndm.ox.ac.uk}

BMJ 2001;322:329-33

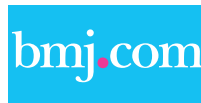

A table giving details of included studies and full references for included and excluded studies is available on the BMJ's website 


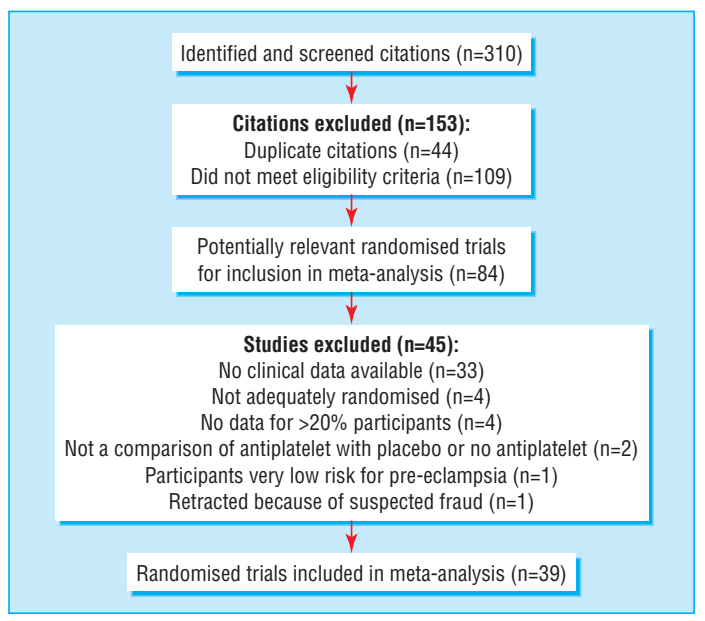

Fig 1 Summary of the systematic review profile

the authors. Data in graphs and figures were included only if the two reviewers independently had the same result. All data entry was double checked manually.

Prespecified outcomes for the women were pre-eclampsia, caesarean section, antepartum haemorrhage, serious maternal morbidity, and rare adverse

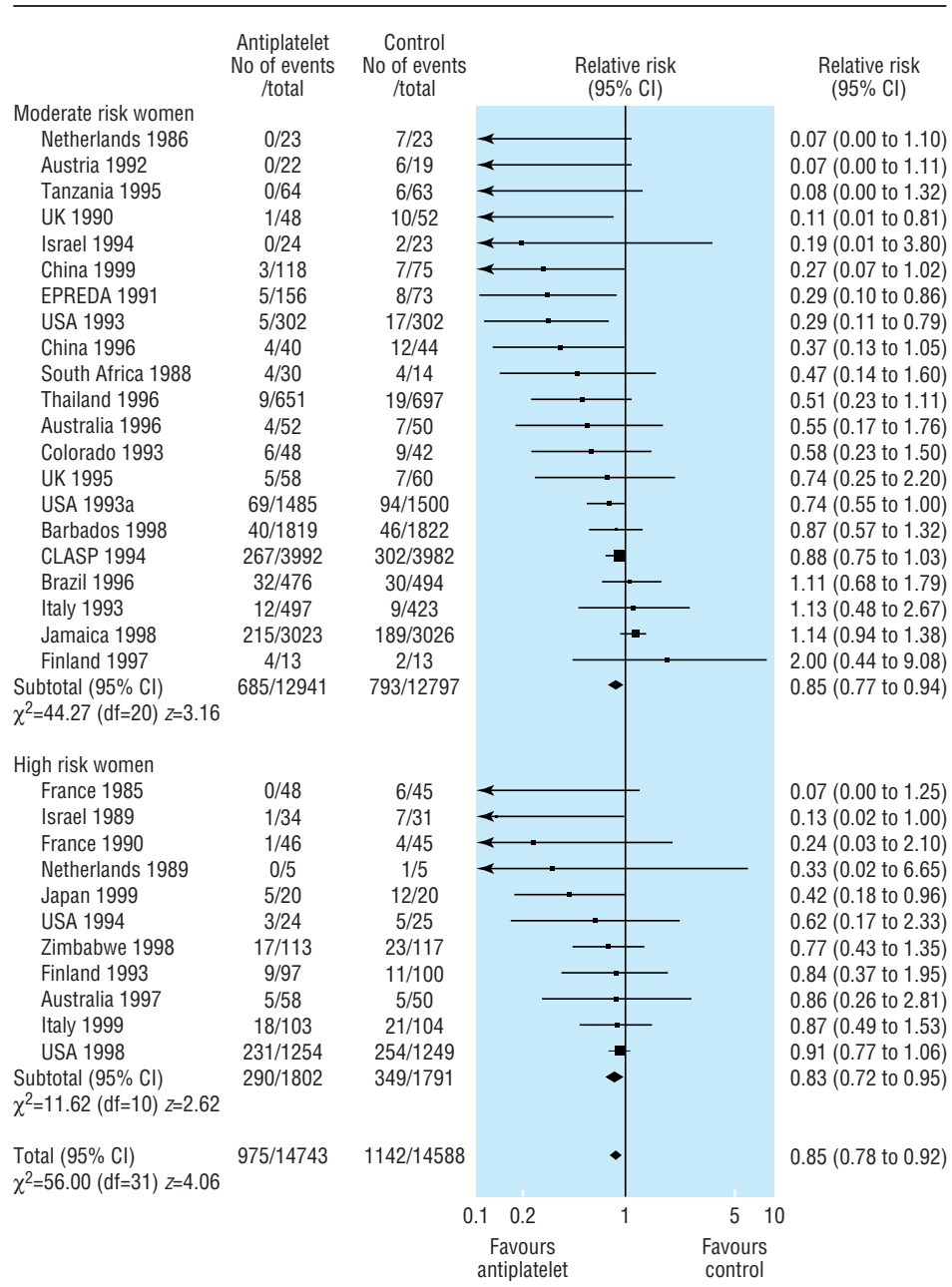

Fig 2 Effect of antiplatelet drugs on pre-eclampsia (subgroups by risk status at trial entry sorted by effect size) events. For the babies, outcomes were death (stillbirth, neonatal, or infant), preterm birth $(<37$ completed weeks), small for gestational age, bleeding episodes, and measures of infant and child development. Assessment of the use of health service resources included antenatal hospital admission, and admission to a special care baby unit.

We analysed the results with Cochrane Collaboration RevMan software (Metaview 3.1) using a fixed effects model. Results are presented as summary relative risk, risk difference, and number needed to treat, which is calculated as $1 /$ risk difference. Graphs were sorted by effect size to assess potential asymmetry in their distribution.

Women were classified into prespecified subgroups based on whether they were at high or moderate risk of developing pre-eclampsia at trial entry, were before or after 20 weeks gestation at trial entry, the dose of aspirin was $\leqslant 75 \mathrm{mg}$ or $>75 \mathrm{mg}$, and the study used a placebo for the control group. High risk of preeclampsia was defined as one or more of previous severe pre-eclampsia, diabetes, chronic hypertension, renal disease, or autoimmune disease. Moderate risk was defined as any other risk factors. When risk was unclear or unspecified, women were classified as moderate risk.

Studies were divided by whether they were published before or after the end of 1994, when the collaborative low dose aspirin study in pregnancy ${ }^{\mathrm{w} 11}$ was published, and classified according to where the main report was published and number of citations. Date recruitment stopped was compared with date and place of publication.

\section{Results}

The search strategy yielded 310 citations. Of these, 153 were excluded; 44 were duplicate citations and 109 did not meet the eligibility criteria. Of the remaining 157 , 99 referred to a total of 39 included trials and 58 referred to 45 excluded studies (fig 1 ).

The 39 included trials recruited 30563 women. Thirteen trials included fewer than 50 women, five included 50-99 women, 13 included 100-999 women, and eight included 1000 or more women. There was a wide range in risk status between women in the different studies and, often, between women in the same trial. One study did not report outcomes separately for treatment and prevention and so we included all women. ${ }^{18}$

Details of the methods, participants, interventions, and outcomes of the included studies are available on the BMJ's website. Most studies compared aspirin alone with placebo (28 802 women). Four studies used a combination of aspirin and dipyridamole compared with control, one used heparin with dipyridamole compared with control, and another compared ozagrel hydrochloride with placebo. Thirteen small trials used $>75 \mathrm{mg}$ aspirin (1264 women). Eight trials did not use a placebo. Details of the 45 excluded studies have been published. ${ }^{15}$ Figure 1 gives the reasons for exclusion.

Quantitative data synthesis

There was good agreement between reviewers for selection and assessment of validity, and discrepancies were quickly resolved. Concealment of allocation was 
graded a for 14 trials, $\mathrm{b}$ for 24 , and c for one study. Data for all outcomes and subgroups are available. ${ }^{15}$

\section{Maternal outcomes}

There was no overall difference in the risk of pregnancy induced hypertension in the 27 trials (18 147 women) reporting this outcome (relative risk $0.97,95 \%$ confidence interval 0.89 to 1.05 ). The $15 \%$ reduction in risk of pre-eclampsia associated with antiplatelet drugs (32 trials, 29331 women; relative risk $0.8595 \%$ confidence interval 0.78 to 0.92 ; risk difference $-0.01,95 \%$ confidence interval -0.017 to -0.006 ; number needed to treat $100,95 \%$ confidence interval 59 to 167) was consistent regardless of risk status (fig 2), dose of aspirin, gestation at trial entry, or use of a placebo. It was greater for women allocated $>75$ $\mathrm{mg}$ aspirin (relative risk $0.35,0.24$ to 0.52 ), but the numbers in this subgroup are small (13 trials, 1264 women).

There were no significant differences between treatment and control groups in the risk of eclampsia (nine trials, 14623 women), maternal death (two trials, 9438 women), caesarean section (17 trials, 25827 women), induction of labour (three trials, 15935 women), antenatal admission (one trial, 6049 women), or placental abruption (12 trials, 22309 women).

\section{Outcomes for babies}

In 23 trials (28 268 women) that reported preterm birth there was a small $(8 \%)$ reduction in the risk of being born too early (relative risk $0.92,0.88$ to 0.97 ; risk difference $-0.014,-0.023$ to -0.005 ; number needed to treat 72, 44 to 200 ; fig 3 ). The size of this reduction was consistent across all subgroups, except those receiving $>75 \mathrm{mg}$ aspirin (557 women; relative risk $0.58,0.38$ to 0.88$)$.

Thirty trials (30 093 women) reported fetal, neonatal, or infant deaths. Overall there was a 14\% reduction in deaths in the antiplatelet group compared with the control group (relative risk $0.86,0.75$ to 0.98 ; risk difference $-0.004,-0.008$ to 0.000 ; number needed to treat 250,125 to $>10000$; fig 4 ).

In 25 trials (20 349 women) there was no clear difference in the risk of small for gestational age births (relative risk $0.92,0.84$ to 1.01 ; fig 5). In two subgroups the difference was significant: women randomised before 20 weeks ( 8401 women, $0.82,0.71$ to 0.96 ) and those given $>75 \mathrm{mg}$ aspirin (934 women, $0.68,0.52$ to $0.88)$.

There were no significant differences between treatment and control groups in the risk of birth weight $<2500 \mathrm{~g}$ ( 4 trials, 7391 women), admission to

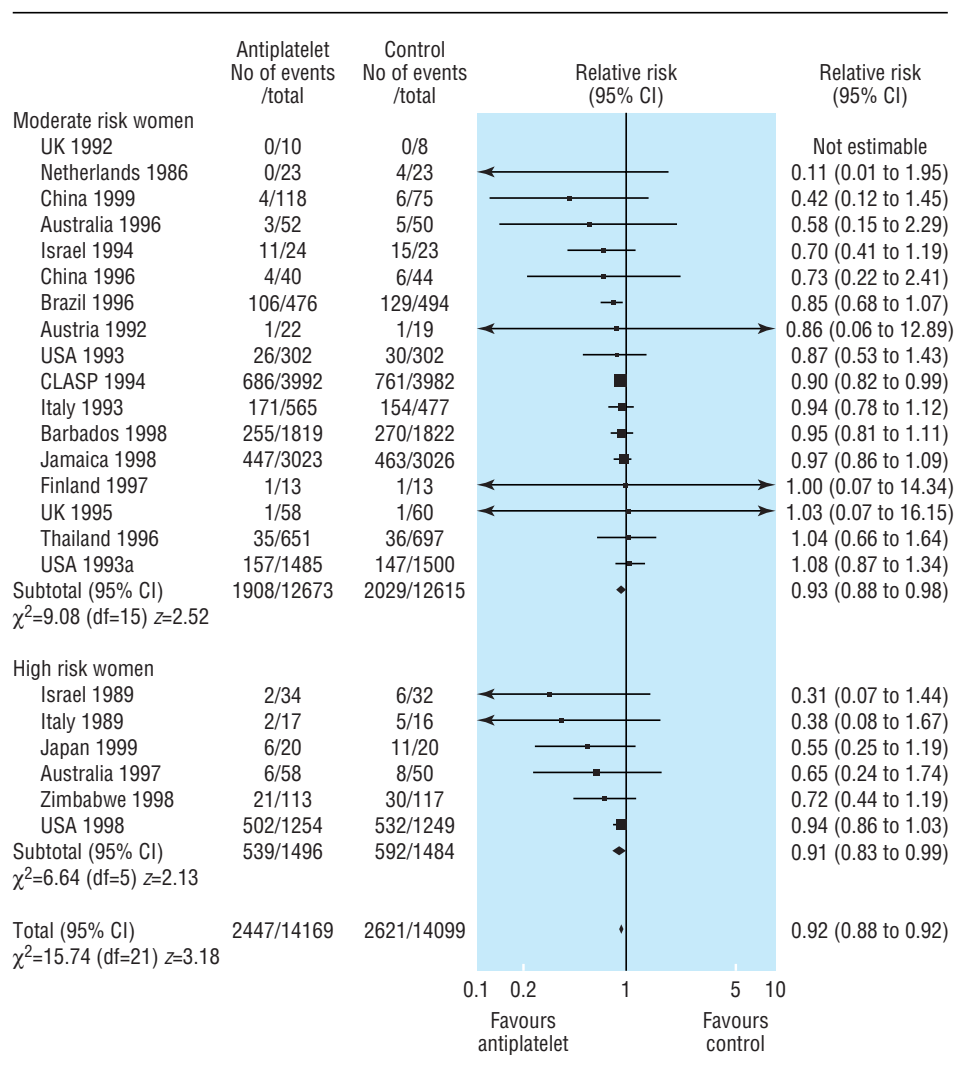

Fig 3 Effect of antiplatelet drugs on preterm delivery (subgroups by risk status at trial entry, sorted by effect size)

special care baby unit (12 trials, 25641 women), intraventricular haemorrhage (eight trials, 22793 women), or other neonatal bleeding (six trials, 23591 women). One large trial assessed child health and development at 12-18 months, no difference was apparent between treatment and control groups. ${ }^{19}$

\section{Potential publication bias}

Twenty three included studies were published before the end of 1994 and 16 after the start of 1995 (table). The early trials were more likely to be published in a high profile general journal $(11 / 23 v 1 / 16)$ and to be available only as an abstract (6/23 $v 1 / 16)$. In comparison, 36 excluded studies were published before the end of 1994 and nine after the start of 1995. Early excluded studies were more likely to be published as an abstract only (12/36 $v 0 / 9)$ and to be known about only through prospective registration $(6 / 36 v 0 / 9)$. Only 18 of the 39 included trials reported when recruitment

Publication factors influencing the accessibility and impact of antiplatelet trials included in systematic review

\begin{tabular}{|c|c|c|c|c|}
\hline & \multicolumn{2}{|c|}{ No $(\%)$ of included trials } & \multicolumn{2}{|c|}{ No $(\%)$ of excluded trials } \\
\hline & $\begin{array}{l}\text { Published before } 1995 \\
(\mathrm{n}=23)\end{array}$ & $\begin{array}{c}\text { Published } 1995 \text { or } \\
\text { later }(n=16)\end{array}$ & $\begin{array}{l}\text { Published before } 1995 \\
\qquad(\mathrm{n}=36)\end{array}$ & $\begin{array}{l}\text { Published } 1995 \text { or } \\
\text { later }(n=9)\end{array}$ \\
\hline Main report in high profile general journal ${ }^{*}$ & $11(48)$ & $1(6)$ & $2(6)$ & - \\
\hline Main report in high profile specialist journal† & $4(17)$ & $4(25)$ & $6(17)$ & $2(22)$ \\
\hline Published as abstract only & $6 \ddagger(26)$ & $1(6)$ & $12(33)$ & - \\
\hline Main report not in English & - & $1(6)$ & $4(11)$ & $2(22)$ \\
\hline$\geqslant 3$ citations/study & $8(35)$ & $3(19)$ & $1(3)$ & - \\
\hline Prospective registration only & - & - & $6(17)$ & - \\
\hline
\end{tabular}

*Lancet, BMJ, New England Journal of Medicine, or JAMA.

†American Journal of Obstetrics and Gynecology, Obstetrics and Gynecology, or British Journal of Obstetrics and Gynaecology.

łIncludes one MD thesis. 
started and stopped, and so assessment of potential bias in the speed with which results were published is not possible.

\section{Discussion}

Our review of data from over 30000 women suggests that antiplatelets drugs are associated with a moderate $(15 \%)$ reduction in the risk of pre-eclampsia, a $14 \%$ reduction in the risk of a stillbirth or neonatal death, and an $8 \%$ reduction in the risk of preterm birth. There was some evidence that there may be greater benefits for women given $>75 \mathrm{mg}$ aspirin, although the numbers of women in the subgroup were small and so there is potential for random error. Also, the risks of this dose may be increased as current data on safety applies largely to $60 \mathrm{mg}$ aspirin.

The relatively large number of subgroups, although pre-specified, means that some of the significant results may reflect the play of chance. In addition, there is potential for misleading bias in analyses for which only a proportion of eligible trials reported the outcome and large numbers of women were missing.

For aspirin to prevent pre-eclampsia it may need to be started well before trophoblast invasion is complete. We presented data before and after 20 weeks, as this is how they are reported in the trials, but there is little evidence of clinically worthwhile differences. The

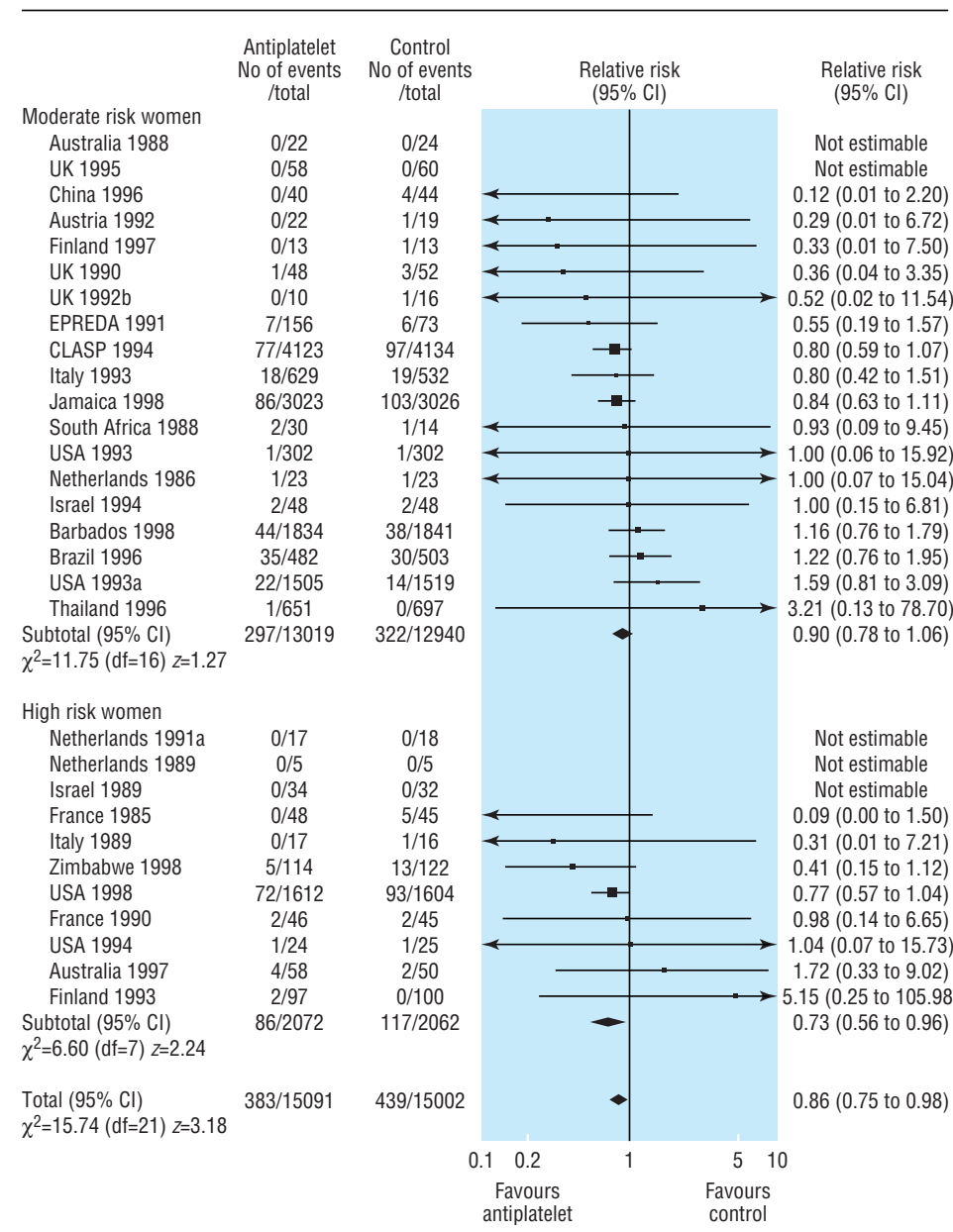

Fig 4 Effect of antiplatelet drugs on stillbirth and neonatal death (subgroups by risk status at trial entry, sorted by effect size)

\section{What is already known on this topic}

Early systematic reviews of antiplatelet drugs, largely low dose aspirin, included only small trials and reported promising reductions in the risk of developing pre-eclampsia and its complications

Subsequent large trials have failed to confirm a large reduction in the risk of pre-eclampsia

Successive meta-analyses have concluded that aspirin is not effective

\section{What this study adds}

Data from over 30000 women show that antiplatelet drugs are associated with a $15 \%$ decrease in the risk of pre-eclampsia

These drugs also have a small effect on the risk of stillbirth, neonatal death, and prematurity

Data from individual women need to be reviewed to identify which women are most likely to benefit, when treatment should be started, and at what dose

crucial time for starting treatment may be before 16 or even 12 weeks.

This review includes women with a wide range of clinical risk. Although this enhances the generalisability of the results, it is not possible to disentangle the effects for women with specific conditions or risk factors. Such an analysis requires data from individual women and is now being planned.

The discrepancy between the results of early metaanalyses of antiplatelet drugs and later large trials has been quoted as an example of a misleading meta-analysis. ${ }^{20}$ One plausible explanation for this discrepancy is publication bias. For example, only four of the 32 included trials that reported pre-eclampsia have a point estimate favouring the control group. Publication bias may be the reason for this lack of negative trials. Eighteen excluded studies are available only through abstracts or prospective registration. These may be missing negative results. An alternative explanation for the discrepancy is a different case mix in small and large trials. ${ }^{21}$ The small trials included only high risk women who really benefited from antiplatelet drugs, whereas in the later large trials these effects were diluted by moderate risk women with little potential for benefit. ${ }^{22}$

\section{Implications}

As most of the evidence relates to aspirin $(<75 \mathrm{mg})$, this is the antiplatelet drug of choice. Data from this review should be made available to pregnant women as well as clinicians and policy makers. As the reductions in risk are moderate, relatively large numbers of women will need to be treated to prevent a single adverse outcome. For example, the point estimate is that 250 women need to be treated to prevent the death of one baby, but the true number could be anywhere between 125 and 10000 women. From a public health perspective, however, even these moderate benefits may be worth while. Starting 
treatment before 12 weeks and using higher doses cannot be recommended until more information is available about safety.

Remaining questions are whether small subgroups of high risk women might have greater benefit and whether earlier treatment or a higher dose would have additional benefits without an increase in adverse effects. These unresolved questions would be most efficiently answered by a review pooling data from individual women in the trials presented here rather than undertaking further trials.

The antiplatelet trialists formed a collaborative group in July 2000. Any new trialists interested in collaboration should contact LD or DH-S. We thank Sonja Henderson, Lynn Hampson, and Claire Winterbottom at the Cochrane Pregnancy and Childbirth Group Coordinating Office for help and support. We also thank the referees and editors of the Cochrane Pregnancy and Childbirth Group for their thoughtful and constructive comments.

Contributors: All authors contributed to developing the protocol. MK wrote the first draft of the protocol, which was modified in discussion with all the authors and following comments from others. MK and LD did the searches and decided on potentially eligible studies. All authors contributed to data extraction. Data were entered by MK, LD, and DH-S. Al authors contributed to checking the data. All authors commented on the final report, which was drafted and revised by LD. LD and DH-S will act as guarantors for the study

Funding: None.

Competing interests: None declared.

1 Gifford RW, August P, Chesley LC, Cunningham G, Ferris TF Lindheimer MD, et al. National high blood pressure education program working group report on high blood pressure in pregnancy. Am J Obstet Gynecol 1990;163:1689-712.

2 World Health Organization International Collaborative Study of Hypertensive Disorders of Pregnancy. Geographic variation in the incidence of hypertension in pregnancy. Am J Obstet Gynecol 1988;158:80-3.

3 Duley L. Maternal mortality associated with hypertensive disorders of pregnancy in Africa, Asia, Latin America and the Caribbean. Br J Obstet Gynaecol 1992;99:547-53.

4 Department of Health, Welsh Office, Scottish Home and Health Department, Department of Health and Social Security. Report of confidential enquiries into maternal deaths in the United Kingdom 1994-1996. London: HMSO, 1998

5 Department of Health. Confidential enquiry into stillbirths and deaths in infancy: 3rd annual report. London: $\mathrm{DoH}, 1996$.

6 Taylor DJ. The epidemiology of hypertension during pregnancy. In Rubin PC, ed. Hypertension in pregnancy. Amsterdam: Elsevier Science, 1988:223-40.

7 Bussolino F, Benedetto C, Massobrio M, Camussi G. Maternal vascular prostacyclin activity in pre-eclampsia. Lancet 1980;ii:702.

8 Collins R. Antiplatelet agents for IUGR and pre-eclampsia. In: Enkin MW, Keirse MJNC, Renfrew MJ, Neilson JP, Crowther C, eds. Pregnancy and childbirth module. In: Cochrane Collaboration: Cochrane Pregnancy and Childbirth Database. Issue 2. Oxford: Update Software, 1995.

9 Imperiale TF, Petrulis AS. A meta-analysis of low-dose aspirin for the prevention of pregnancy-induced hypertensive disease. JAMA 1991;266:260-4

10 Rey E, Derderian F. Efficacite de l'aspirine a faible dose au cours de al grossesse en fonction des facteurs de risque maternels et foetaux. $J S$ Obstet Gynecol C 1996;18:51-60.

11 Sanchez-Ramos L, Wears R, Del Valle GO, Gaudier FL, Adair D. Low dose aspirin for the prevention of pregnancy-induced hypertension: a meta-analysis. Am J Obstet Gynecol 1994;170:408.

12 Sharts-Engel NC. Aspirin for prevention of pregnancy-induced hypertension. Am J Child Nurs 1992;17:168.

13 Leitich H, Egarter C, Husslein P, Kaider A, Schemper M. A meta-analysis of low dose aspirin for prevention of intrauterine growth retardation. $\mathrm{Br}$ J Obstet Gynaecol 1997;104:450-9.

14 Broughton Pipkin F, Crowther C, de Swiet M, Duley L, Judd A, Lilford RJ et al. Where next for prophylaxis against pre-eclampsia? $\mathrm{Br} J$ Obstel Gynaecol 1996;103:603-7.

15 Knight M, Duley L, Henderson-Smart D, King J. Antiplatelet agents for preventing and treating pre-eclampsia. In: Cochrane Collaboration: Cochrane Library. Issue 3. Oxford: Update Software, 2000.

16 Cochrane Controlled Trials Register. In: Cochrane Collaboration: Cochrane Library. Issue 1. Oxford: Update Software, 1999.

17 Mulrow CD, Oxman AD, eds. Cochrane collaboration handbook. In: Cochrane Collaboration: Cochrane Library. Issue 1. Oxford: Update Software, 1999.

18 Italian Study of Aspirin in Pregnancy. Low-dose aspirin in prevention and treatment of intrauterine growth retardation and pregnancy-induced hypertension. Lancet 1993;341:396-400.

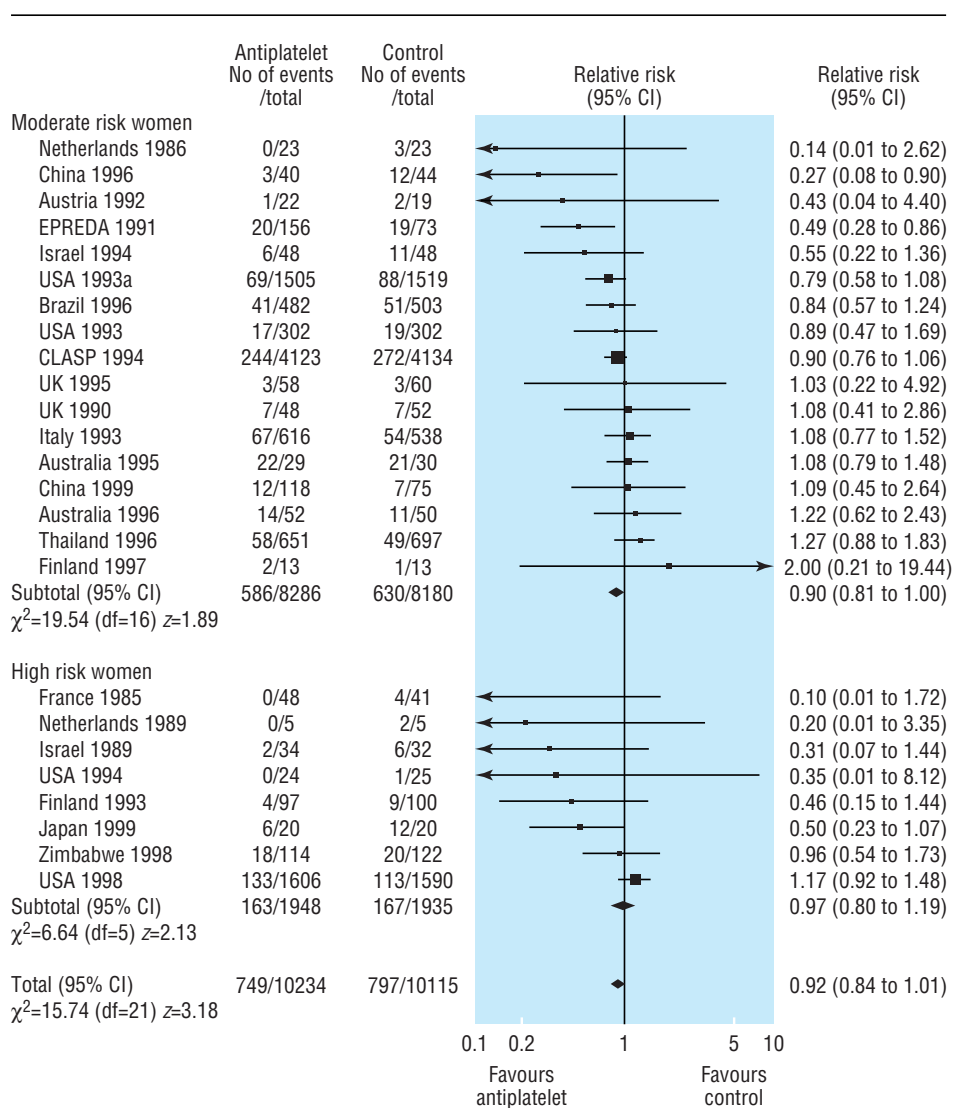

Fig 5 Effect of antiplatelet drugs on small for gestational age babies (subgroups by risk status at trial entry, sorted by effect size)

19 CLASP Collaborative Group. Low dose aspirin in pregnancy and early childhood development: follow up of the collaborative low dose aspirin study in pregnancy. Br J Obstet Gynaecol 1994;102:861-8.

20 Egger M, Davey Smith G. Misleading meta-analysis. BMJ 1995;310:752-4

21 Egger M, Davey Smith G, Schneider M, Minder C. Bias in meta-analysis detected by a simple graphical test. BMJ 1997;315:629-34.

22 Grant JM. Multicentre trials in obstetrics and gynaecology. Smalle explanatory trials are required. Br J Obstet Gynaecol 1996;103:599-602.

(Accepted 9 November 2000)

\section{Endpiece \\ A local celebrity}

It was an element in Doctor Sloper's reputation that his learning and his skill were very evenly balanced; he was what you might call a scholarly doctor, and yet there was nothing abstract in his remedies-he always ordered you to take something. Though he was felt to be extremely thorough, he was not uncomfortably theoretic; and if he sometimes explained matters rather more minutely than might seem of use to the patient, he never went so far (like some practitioners one had heard of) as to trust to the explanation alone, but always left behind him an inscrutable prescription. There were some doctors that left the prescription without offering any explanation at all; and he did not belong to that class either, which was after all the most vulgar. It will be seen that I am describing a clever man: and this is really the reason why Doctor Sloper had become a local celebrity.

Henry James, Washington Square, 1880 Submitted by D R Laurence, emeritus professor of pharmacology and therapeutics, London 